\title{
Non-ideal Models and Simulation of Boost Converters Operating in DCM
}

\author{
Guang-jun Xie, Senior Member, IACSIT, Hai-bin Fang and Xin Cheng
}

\begin{abstract}
Various aspects for the averaged modelling of the Boost converters operating in the discontinuous conduction mode (DCM) are studied. The parasitics of the Boost converter are also considered. A duty ratio constraint that defines the diode conduction interval is identified to be the key for accurate prediction of high-frequency behaviour. A new duty-ratio constraint is proposed that leads to the full-order averaged models of DCM converters. Numerical analyses and experimental measurements confirm that the new models correctly predict the small-signal responses up to the half of switching frequency and are more accurate than previous models. Moreover, the analytical results are included to show the origin of the high-frequency pole in DCM operation and to explain why the full-order model is capable of accurately predicting it.
\end{abstract}

Index Terms-Discontinuous Conduction Mode, Boost Converters, Duty Ratio Constraint

\section{INTRODUCTION}

For the high stability and efficiency of DC-DC switching converter, it has been applied to the fields of mobile communications, industrial equipment, home appliances, and so on. The analyses and design of the switch converter already becomes the key points of the research. However, the pulse-width modulation (PWM) switching converters are strongly non-linear time-varying circuits. It is difficult to accurately find their analytical solutions. So, to find a suitable model for simulation of the DC-DC switching power supply design is an important role.

DC-DC converter has two basic operating modes, namely, continuous conduction mode (CCM) and discontinuous conduction mode. In this paper, the Boost DC-DC converter is taken for example, the average circuit method is used to study its non-ideal model operating in DCM. In the proposed methodology, the so-called duty-ratio constraint is used to make improvement in the model. Thereafter, the calculated transfer-functions for steady-state and dynamic small-signal characteristics are also obtained.

The DCM operation of PWM converters differs from CCM operation by an additional time interval in each

Manuscript received December 5, 2009. This work was supported by the Excellent Young Scientist Foundation of Anhui province under Grant No. 06042086.

Gang-jun Xie is with School of Electronic Science and Applied Physics, Hefei University of Technology, Hefei 230009, Anhui, China (corresponding author, e-mail: gjxie8005@hfut.edu.cn).

Hai-bin Fang is with School of Electronic Science and Applied Physics, Hefei University of Technology, Hefei 230009, Anhui, China.

Xin Cheng is with School of Electronic Science and Applied Physics, Hefei University of Technology, Hefei 230009, Anhui, China. switching cycle during which an inductor current is clamped to zero (or a constant when there are multiple energy storage elements). Therefore, the DCM operation typically occurs in the DC-DC converters at light load which means a low load current. Many efforts have been made in the past decades to model DCM converters[1-6], these models fall into two categories, i.e. linear models and non-linear models. Non-linear model can be also called full-order averaged model [7] which shows great improvement over the linear model. Conduction losses based on the switch-cell and parasitics are included in the non-linear model.

\section{DUTY-RATIO CONSTRAINT}

The inductor current waveform of Boost converter in DCM is shown in Fig. 1.

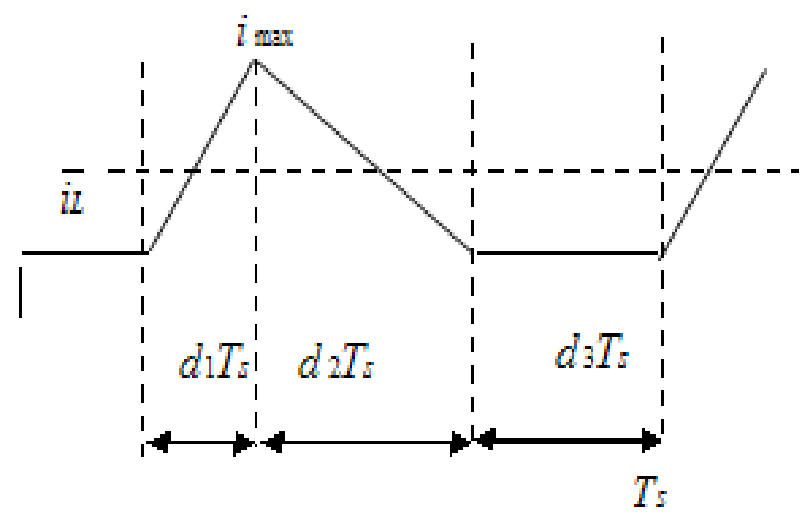

Fig.1. The inductor current waveform of Boost converter in DCM

A duty-ratio constraint defining the dependency of $d_{2}$ on other variables is identified to be the key to accurately predict the high-frequency behavior. In the conventional state-space averaging method, a volt-second balance relation of the inductor is used to define the duty-ratio constraint [8]. For the Boost topology, volt-second balance over a switching cycle implies

$$
d_{2}=\frac{v_{i}}{v_{c}-v_{i}} d_{1}
$$

In the conventional state-space averaging method, using this expression can derive average state equation of ideal converter.

$$
\begin{aligned}
& \frac{d \bar{i}_{L}}{d t}=0 \\
& \frac{d \bar{v}_{c}}{d t}=\frac{v_{i}}{\bar{v}_{c}} \frac{\bar{i}_{L}}{C}-\frac{\bar{v}_{c}}{R C}
\end{aligned}
$$

In equation (2), $\bar{i}_{L}$ is a linear function, however, in reality 
$\bar{i}_{L}$ is a non-linear function, $d \bar{i}_{L} / d t$ is relevant with the state variables, $\bar{i}_{L}$ and $\bar{v}_{c}$. Equation (1) yields a degenerate model where the dynamics of the inductor current disappear. Since the inductor current is no longer a state variable, this averaged model fails to predict the high-frequency behavior of converters.

In the following, a new definition of $d_{2}$ is derived as

$$
\begin{aligned}
& \bar{i}_{L}=\frac{i_{\max }}{2}\left(d_{1}+d_{2}\right) \\
& \text { And } i_{\text {max }}=\frac{v_{i}}{L} d_{1} T_{S}
\end{aligned}
$$

Taking equation (5) into equation (4) can get the solution

$$
d_{2}=\frac{2 L \bar{i}_{L}}{d_{1} T_{s} v_{i}}
$$

Comparing with equation (1), the equation (6) will derive a full order averaged model which can correctly predict the high frequency responses as well as dc and low frequency behavior of DCM converters.

\section{New Non-Ideal Model For Boost Converter}

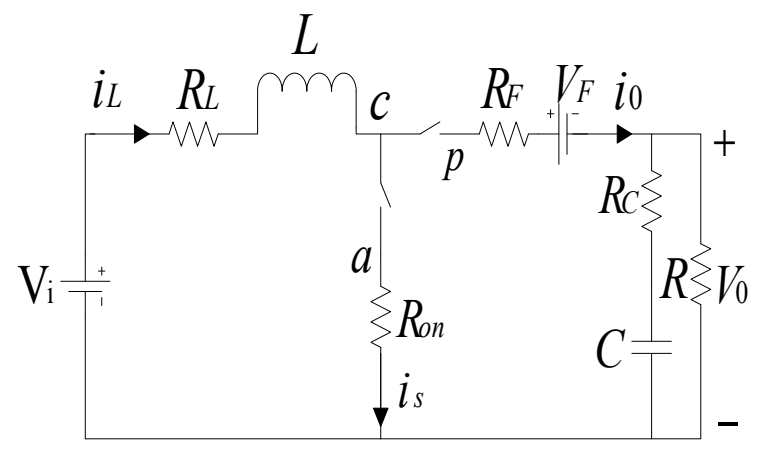

Fig.2. The non-ideal Boost converter

An essential step in constructing the dynamic averaged-switch model is to identify the three-terminal network of the switched-inductor cell as shown in Fig. 2. Here, the parasitics are decoupled to form an ideal part of the switched-inductor cell (a, p and c) for which a corrected full-order model based on the fast-state dynamics is considered. Then, the parasitics are placed outside the averaged cell and appropriately modified to account for energy dissipation.

Two switching inductance parasitics are equalled to the slip road under the principle of conservation of energy, and then the average model of switching elements method is used, the switching elements are replaced by a current-controlled current source, the passive switching elements are replaced by a voltage-controlled voltage source, shown in Fig. 3 .

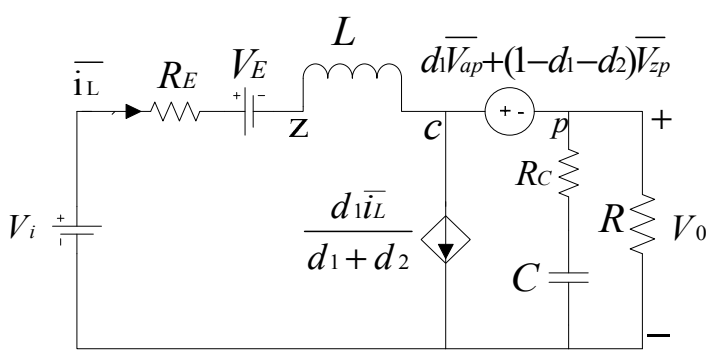

Fig.3. The large-signal averaging model of non-ideal Boost converter in DCM

$$
\begin{gathered}
R_{E}=\frac{4}{3\left(D_{1}+D_{2}\right)}\left(R_{L}+\frac{D_{1} R_{o n}}{D_{1}+D_{2}}+\frac{D_{2} R_{F}}{D_{1}+D_{2}}\right) \\
V_{E}=\frac{D_{2}}{D_{1}+D_{2}} V_{F}
\end{gathered}
$$

Considering both the $\mathrm{dc}$ and the small-signal ac components of switch current, duty cycle, and switch voltage, there are $d_{1}=D_{1}+\hat{d}_{1} \quad, \quad \bar{i}_{L}=I_{L}+\hat{i}_{L} \quad$, $v_{i}=V_{i}+\hat{v}_{i} \quad, \quad \bar{v}_{a p}=V_{a p}+\hat{v}_{a p} \quad, \quad \bar{v}_{z p}=V_{z p}+\hat{v}_{z p} \quad$. For $\left|\hat{d}_{1}\right| \ll<D_{1}, \quad\left|\hat{i}_{L}\right| \ll<I_{L}, \quad\left|\hat{v}_{i}\right| \ll<V_{i}, \quad\left|\hat{v}_{a p}\right|<<V_{a p}$, $\left|\hat{v}_{z p}\right|<<V_{z p}$, the following results can be deduced.

$$
\begin{gathered}
\frac{d_{1} \overline{i_{L}}}{d_{1}+d_{2}}=\frac{d_{1}^{2} T_{S} v_{i}}{2 L}=\frac{\left(D_{1}+\hat{d}_{1}\right)^{2}\left(V_{i}+\hat{v}_{i}\right) T_{S}}{2 L} \\
=\frac{D_{1}^{2} T_{S}}{2 L} \hat{v}_{i}+\frac{D_{1} V_{i} T_{S}}{L} \hat{d}_{1}=a_{1} \hat{v}_{i}+a_{2} \hat{d}_{1} \\
d_{1} \bar{v}_{a}+\left(1-d_{1}-d_{2}\right) \bar{v}_{z} p \\
=\left[1+\frac{2 L\left(V_{E}+V_{O}+I_{L} R_{E}\right) I_{L}}{D_{1} V_{i}^{2} T_{S}}-\frac{4 L I_{L}}{D_{1} V_{i} T_{S}}\right] \hat{v}_{i} \\
+\left[-V_{O}+\frac{2 L\left(V_{i}-V_{E}-V_{O}-I_{L} R_{E}\right) I_{L}}{D_{1}^{2} V_{i} T_{S}}\right] \hat{d}_{1} \\
+\left[-R_{E}+\frac{2 L R_{E} I_{L}}{D_{1} V_{i} T_{S}}-\frac{2 L\left(V_{i}-V_{E}-V_{O}-I_{L} R_{E}\right)}{D_{1} V_{i} T_{S}}\right] \hat{i}_{L} \\
+\left[-D_{1}-1+\frac{2 L I_{L}}{D_{1} V_{i} T_{S}}\right] \hat{v}_{o}=b_{1} \hat{v}_{i}+b_{2} \hat{d}_{1}+b_{3} \hat{i}_{L}+b_{4} \hat{v}_{o}
\end{gathered}
$$

Based on the above, the small-signal model is given as follow,

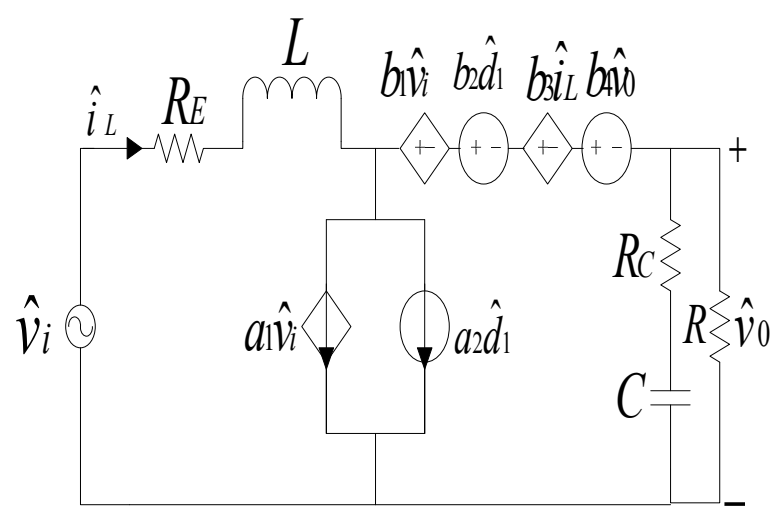

Fig.4. The small-signal model of Boost converter in DCM

(1)The input-to-output transfer function is 


$$
\begin{gathered}
G_{v i}(s)=\left.\frac{\hat{v}_{o}(s)}{\hat{v}_{i}(s)}\right|_{\hat{d} 1(s)=0} \\
=\frac{1-b_{1}-a_{1}\left(s L+R_{E}+b_{3}\right)}{1+b_{4}+\frac{\left(s L+R_{E}+b_{3}\right)(s R C C+s R C+1)}{R(s R C C+1)}} \\
=\frac{k_{1 S}+t_{1}}{t_{0}+\frac{\left(k_{2 S}+t_{2}\right)\left(k_{3 S}+t_{3}\right)}{k_{4 S}+t_{4}}} \\
=\frac{k_{1} k_{4} S^{2}+k_{1} t_{4 S}+k_{4} t_{1 S}+t_{1} t_{4}}{k_{2} k_{3} s^{2}+k_{2} t_{3} s+k_{3} t_{2} s+k_{4} t_{0} s+t_{2} t_{3}+t_{0} t_{4}} \\
=\frac{t_{1} t_{4}}{t_{2} t_{3}+t_{0} t_{4}} \cdot \frac{\left(1+s / w_{21}\right)\left(1+s / w_{z 2}\right)}{1+\frac{s}{Q w_{0}}+\left(\frac{s}{w_{0}}\right)^{2}}
\end{gathered}
$$

(2) The control-to-output transfer function is

$$
\begin{array}{r}
G_{v d}(s)=\left.\frac{\hat{v}_{o}(s)}{\hat{d}_{1}(s)}\right|_{\hat{v}_{i}(s)=0} \\
=\frac{t_{4} t_{5}}{t_{2} t_{3}+t_{0} t_{4}} \cdot \frac{\left(1+s / w_{z 2}\right)\left(1-s / w_{z 3}\right)}{1+\frac{s}{Q w_{0}}+\left(\frac{s}{w_{0}}\right)^{2}}
\end{array}
$$

The control-to-output transfer function includes the right-half plane (RHP) zero $w_{z 3}$, in low-frequency it can be neglected, but in high-frequency it makes the phase of $G_{v d}(s)$ increased $-90^{\circ}$. As the Fig. 6(b) shows, the new averaged model gives the most accurate response compared to detailed simulation.

(3)The converter input impedance $Z(s)$ is

$$
\begin{aligned}
& Z(s)=\left.\frac{\hat{v}_{i}(s)}{\hat{i}_{i}(s)}\right|_{\hat{d}_{1}(s)=0}=\left.\frac{\hat{v}_{i}(s)}{\hat{i}_{L}(s)}\right|_{\hat{d}_{1}(s)=0} \\
= & \frac{t_{2} t_{3}+t_{0} t_{4}}{t_{3} t_{7}+t_{4} t_{6}} \cdot \frac{1+\frac{s}{Q w_{0}}+\left(\frac{s}{w_{0}}\right)^{2}}{\left(1+s / w_{p}\right)}
\end{aligned}
$$

(4)The converter output impedance $Z_{\text {out }}(s)$ is

$$
\begin{array}{r}
Z_{\text {out }}(s)=\left.\frac{\hat{v}_{o}(s)}{\hat{i}_{\text {out }}(s)}\right|_{\hat{v}_{i}(s)=0, \hat{d}_{1}(s)=0} \\
=\frac{t_{2 t_{4}}}{t_{2} t_{3}+t_{0} t_{4}} \cdot \frac{\left(1+s / w_{z 2}\right)\left(1+s / w_{z 4}\right)}{1+\frac{s}{Q w_{0}}+\left(\frac{s}{w_{0}}\right)^{2}}
\end{array}
$$

\section{SimULATION AND ANALYSIS}

In this paper, the Matlab/Simulink is chosen as the simulation tool. The parameters are, $V_{i}=5 \mathrm{~V}, V_{o}=10 \mathrm{~V}$, $I_{o}=0.2 \mathrm{~A}, R=50 \Omega, L=19.2 \mu \mathrm{H}, R_{L}=3.6 \mathrm{~m} \Omega$, $C=1000 \mu \mathrm{F}, R_{C}=2 \mathrm{~m} \Omega, R_{o n}=10 \mathrm{~m} \Omega, R_{F}=1 \mathrm{~m} \Omega$, $V_{F}=0.4 \mathrm{~V}, f_{s}=50 \mathrm{kHz}$.
Taking $D_{2}=\frac{2 L I_{L}}{D_{1} T_{s} V_{i}}-D_{1}, I_{L}=\frac{I_{o}}{D_{2}}\left(D_{1}+D_{2}\right)$ into equation (11)-(14), specific values can be calculated. The magnitude and phase of the corresponding transfer-functions are plotted in Fig. 6, in which, I, II, III correspond to the following conditions respectively, I. $R_{E} \neq 0, R_{C} \neq 0$, $V_{F} \neq 0, d_{2}=\frac{2 L \overline{i_{L}}}{d_{1} T_{s} v_{i}}-d_{1}$, the parasitics of converter and the definition of $d_{2}$ in corrected full-order model are considered. II. $R_{E} \neq 0, R_{C} \neq 0, V_{F} \neq 0$, $d_{2}=\frac{v_{i}}{v_{o}-v_{i}} \cdot d_{1}$, Taking into account the parasitics, but the duty-ratio constraint is not considered. III. $R_{E}=0, R_{C}=0$, $V_{F}=0, d_{2}=\frac{v_{i}}{v_{o}-v_{i}} \cdot d_{1}$, for the ideal model.

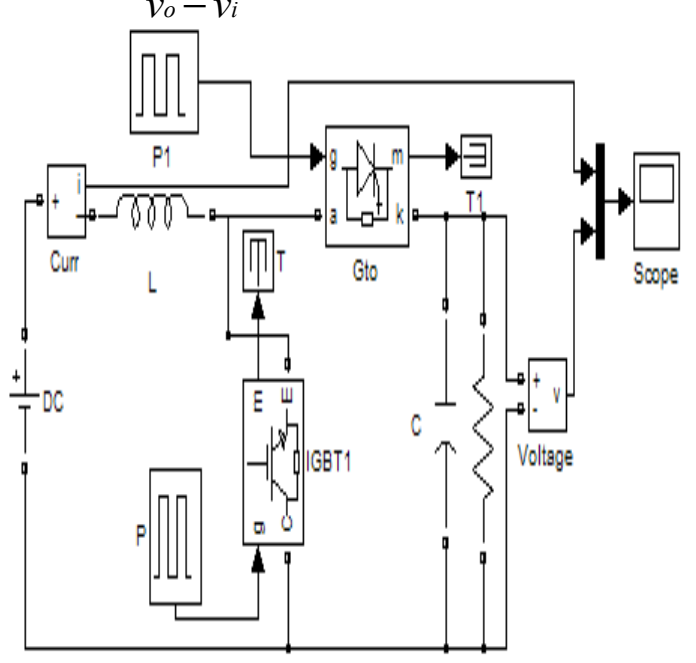

Fig.5. The model of Boost converter in Matlab/Power System Blockset

To get accurate and repeatable measurement points, In Fig. 5 , the hardware prototype is built in Matlab/Power System Blockset, using the FFT utility to extract the necessary magnitude and phase information.

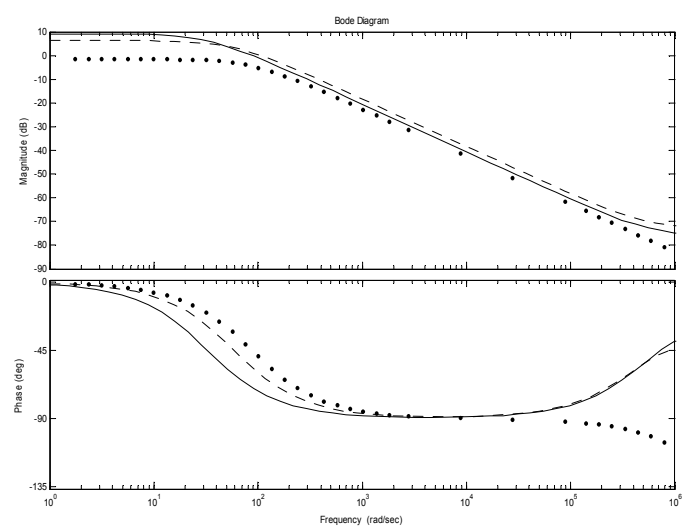

(a) $G_{v i}(s)$ 


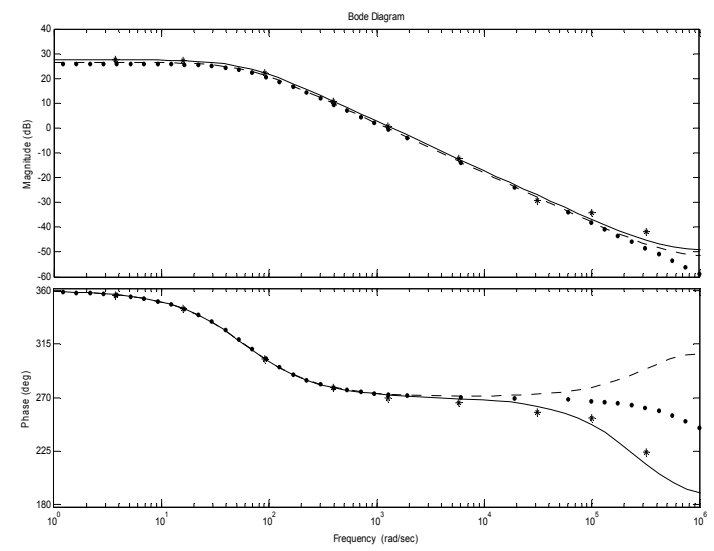

(b) $G_{v d}(s)$

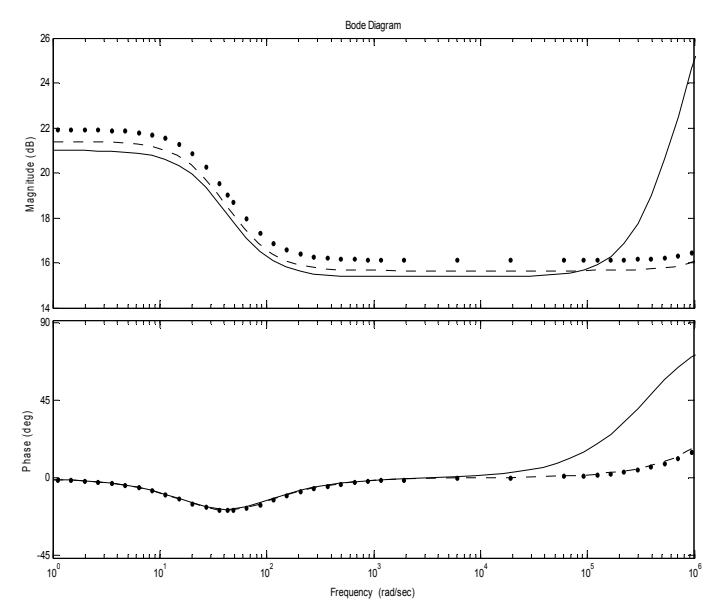

(c) $Z(s)$

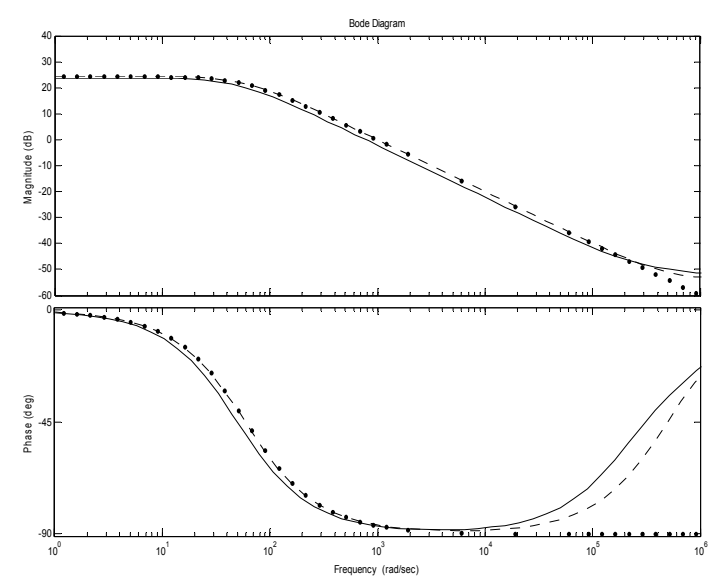

(d) $Z_{\text {out }}(s)$

Fig.6. The magnitude and phase information of the transfer-functions for Boost converter (in which, the solid line, dashed line and dash-dot line, correspond to I, II, III respectively, for example, the $\left(^{*}\right)$ marks in (b) show the hardware measurement)

Suppose as expected, the model proposed in this paper matches the hardware prototype more accurately than the other two previous models. The new model correctly predicts the small-signal responses up to one-half of the switching frequency. Considering the parasitics can more accurately reflect the features of actual converter, it is necessary to consider the parasitics for modeling. A new duty-ratio constraint is proposed, based on formal averaging, which leads to circuit averaged models that are different from previous ones. Numerical simulation and experimental measurement of Boost converter confirm that the new model correctly predicts the small-signal response.

\section{CONCLUSION}

In this paper, a general approach has been presented to generate the circuit averaged models of DC-DC converters operating in DCM. The proposed method relies on a duty-ratio constraint and the parasitics of circuit elements are considered. It accurately captures the high-frequency converter dynamics. The proposed model has been verified with a hardware prototype and has shown an improvement over several existing models.

\section{REFERENCES}

[1] Reatti A, Kazimierczuk M K. Small-signal model of PWM converters for discontinuous conduction mode and its application for Boost converter[J]. IEEE Trans. Circuits and Systems, Vol. 50, No. 1, 65-73, 2003.

[2] Sun J. Unified averaged switch models for stability analysis of large distributed power systems[C]. Proc. APEC, 249-255, 2000.

[3] Prakash B, Prakash S. Analysis of high DC bus voltage stress in the design of single stage single switch switch mode rectifier[J]. IEEE Trans. Power Electron, Vol. 2, No. 20, 505-511, 2005.

[4] Hong Liu, Sheng-gui Tao. The methods of simulation of Boost converter based on MATLAB[J]. Communication Power Supply Technology, Vol. 4, No. 21, 22-24, 2004.

[5] Davoudi A, Jatskevich J, Rybel T D. Numerical state-space average-value modeling of PWM DC-DC converters operating in DCM and CCM[J]. IEEE Trans. Power Electron, Vol. 21, No. 4, 1003-1012, 2006.

[6] Wen-si Cao, Yu-xia Yang. Simulation analyses of Boost converter based on state-space averaging principle [J]. Journal of System Simulation, Vol. 19, No. 6, 1329-1334, 2007.

[7] Xin Cheng, Guang-jun Xie. Full Order Models and Simulation of Boost Converters Operating in DCM[C]. Proc. ICECT, 632-635, 2009.

[8] Sun J, Mitchell D M, Greuel M F. Averaged modeling of PWM converters operating in discontinuous conduction mode[J]. IEEE Trans. Power Electron, Vol. 16, No. 4, 482-492, 2001.

Guang-jun Xie received the B.S. degree and the M.S. degree from Hefei University of Technology, in 1992 and 1995, respectively. He received the Ph.D. degree from University of Science and Technology of China in 2002. He is now a Professor of Hefei University of Technology, and his research interests include IC design, computational intelligence. 